Logik für Philosophen 


\section{Logik \\ für Philosophen}

Von

Prof. Dr. Arnold Oberschelp

Christian-Albrechts-Universität Kiel

Zweite, verbesserte Auflage

Verlag J. B. Metzler Stuttgart · Weimar 
Die Deutsche Bibliothek - CIP - Einheitsaufnahme

Oberschelp, Arnold:

Logik für Philosophen / von Arnold Oberschelp. -

2., verbesserte Aufl. - Stuttgart ; Weimar : Metzler 1997

ISBN 978-3-476-01545-7

NE:. Oberschelp, Arnold

ISBN 978-3-476-01545-7

ISBN 978-3-476-03628-5 (eBook)

DOI 10.1007/978-3-476-03628-5

Dieses Werk einschließlich aller seiner Teile ist urheberrechtlich geschützt. Jede Verwertung außerhalb der engen Grenzen des Urheberrechtsgesetzes ist ohne Zustimmung des Verlages unzulässig und strafbar. Das gilt insbesondere für Vervielfältigungen, Übersetzungen, Mikroverfilmungen und die Einspeicherung und Verarbeitung in elektronischen Systemen.

(C) 1997 Springer-Verlag GmbH Deutschland Ursprünglich erschienen bei J. B. Metzlersche Verlagsbuchhandlung und Carl Ernst Poeschel Verlag GmbH Stuttgart 1997 


\section{Vorwort}

Die Grundkonzeption dieses Buches ist es, eine Einführung in die wichtigsten Begriffe und Sätze der Aussagenlogik und Prädikatenlogik zu geben, die ohne Verzicht auf Strenge auch für Leser zugänglich sein soll, die der Mathematik ferner stehen. Dabei wird versucht, die historischen Zusammenhänge und den grundlagentheoretischen Hintergrund deutlich werden zu lassen, sowie den allgemeinen sprachtheoretischen Aspekt der Logik herauszuarbeiten. Darüber hinaus sollen weitere philosophisch relevante Logiksysteme angesprochen werden.

Die Aussagenlogik dient dazu, in einem einfachen Fall den Aufbau eines logischen Systems mit Syntax, Semantik und der Definition logischer Begriffe vorzuführen. Als Hilfsmittel für die Bearbeitung logischer Fragen wird das Wahrheitstafelverfahren entwickelt.

Die Prädikatenlogik nimmt in diesem Buch die zentrale Stelle ein. Um eine stoffliche Entlastung zu erreichen, wird die einfache Version ohne Funktionszeichen behandelt. Das ist vertretbar, da später in der Klassenlogik ohnehin Funktionen betrachtet werden, und zwar sogar unter Einschluß partieller Funktionen. Ferner wird der Beweis des Vollständigkeitssatzes, der ja in vielen Büchern dargestellt ist, nur skizziert. Es wird jedoch wegen der Bedeutung für die Anwendungen die Prädikatenlogik von vornherein in mehrsortiger Form entwickelt. Der ausführlich behandelte Ableitungskalkül arbeitet mit Annahmen, die in jeder Beweiszeile genannt werden müssen. Dabei werden auch die praktisch wichtigen, aber oft vernachlässigten Ersetzungsschlüsse besprochen. Für die Substitution wird eine eigene Regel formuliert, statt Substitutionen, wie es sonst meist geschieht, in die Quantorenregeln einzuarbeiten. Das erweist sich insbesondere im letzten Kapitel als nützlich.

Eine Besonderheit dieses Buches ist der Abriß einer Klassenlogik, die die Prädikatenlogik erweitert und auch die logische Theorie der Relationen und Funktionen und die Kennzeichnungslogik umfaßt. Die Klassenlogik wird in einer typenfreien Sprache mit Angabe eines vollständigen Ableitungskalküls entwickelt. Sie ist auf eine präzise Semantik gegründet und enthält keinerlei Antinomien.

Zum Abschluß wird ein Ausblick auf die Modallogik und weitere philosophisch relevante Logiksysteme gegeben.

Dieses Buch ist aus Vorlesungen zur Einführung in die Logik hervorgegangen, die ich seit 1974 regelmäßig in Kiel für Philosophen und Studierende nichtmathematischer Fächer gehalten und ständig fortentwickelt habe. $\mathrm{Zu}$ den Vorläufern des Buches gehören, beginnend mit einem handschriftlichen Skript von 1977, verschiedene Vorlesungsausarbeitungen, die auch über verschiedene Auflagen hinweg fortentwickelt wurden. Die letzte Fassung ist für die Buchveröffentlichung abermals überarbeitet und umgearbeitet worden, insbesondere im Kapitel über die Klassenlogik. 
Ich danke allen Mitarbeiten, die mir bei der Erstellung des Manuskriptes geholfen haben. Regina Hornauer schrieb unermüdlich und sorgfältig viele Texte. Torsten Domrös, Detlef Graff, Joachim Gut, Volker Kontze, Lucas Peña, Frank Reitmaier, Detlef Romig, Philipp Rothmaler und Martin Schumann haben in verschiedenen Stadien der Entstehung sorgfältig Korrektur gelesen und manche Anregung gegeben, die aufgegriffen werden konnte.

Kiel, Januar 1992

Arnold Oberschelp

In der zweiten Auflage wurden alle bekannt gewordenen Druckfehler berichtigt und einige Verbesserungen am Text vorgenommen. Ich danke sehr für alle dazu dienlichen Hinweise.

Kiel, Januar 1997

A.O. 


\section{Inhaltsverzeichnis}

§ $0 . \quad$ Historische Vorbemerkung $\quad 9$

\section{Kapitel I Einleitung}

§ 1. Erste Abgrenzung der Logik 17

§ 2. Aussagen 19

§ 3. Logische Folgerungen 24

$\S 4$. Weitere logische Begriffe 28

§ 5. Logische Konstanten 30

§ 6. Nichtlogische Konstanten und Variablen 32

$\S 7 . \quad$ Bemerkungen zur Metatheorie 36

\section{Kapitel II Aussagenlogik}

§ 8. Die formalen Sprachen der Aussagenlogik 39

§ 9. Die Semantik der Aussagenlogik 43

§10. Logische Begriffe für die Aussagenlogik 47

$\S 11$. Das Wahrheitstafelverfahren $\quad 51$

§12. Liste von Tautologien $\quad 56$

$\S 13$. Andere Junktoren 58

$\S 14 . \quad$ Normalformen $\quad 60$

$\S 15$. Vollständige Junktorenmengen 65

Kapitel III Prädikatenlogik

§16. Übergang zur Prädikatenlogik $\quad 67$

§17. Die formalen Sprachen der Prädikatenlogik $\quad 72$

§18. Die Semantik der Prädikatenlogik 81

$\S 19$. Formalisieren und Verbalisieren 93

§20. Logische Begriffe für die Prädikatenlogik 104

$\S 21 . \quad$ Das ABC der Prädikatenlogik $\quad 114$

§22. Syllogismen 118

§23. Ableitbarkeit (aussagenlogische Regeln) 127

§24. Ableitbarkeit (Quantorenregeln) 142

§25. Substitutionen 149

§26. Ersetzungen 156

§27. Ableitbarkeit (Identitätsregeln) 162

§28. Andere Quantoren 167

§29. Vollständigkeit und Unentscheidbarkeit 169

§30. Aufhebung der Sortenbeschränkungen 175 


\section{Kapitel IV Klassenlogik}

§31. Vorbereitung der Klassenlogik 179

$\begin{array}{ll}\text { §32. Klassen } & 187\end{array}$

§33. Relationen 194

§34. Kennzeichnungen 201

§35. Funktionen 206

§36. Die Semantik der Klassenlogik 210

Anhang

$\begin{array}{ll}\text { §37. Ausblick auf weitere Logiksysteme } & 217\end{array}$

Literaturverzeichnis $\quad 225$

Verzeichnis von Symbolen und Schreibweisen $\quad 228$

Personenverzeichnis 231

$\begin{array}{ll}\text { Sachverzeichnis } & 233\end{array}$ 


\section{§. Historische Vorbemerkung}

Seit den Anfängen der Wissenschaft ist logisches Schließen praktiziert worden, doch ist damit noch nicht die Logik als eine wissenschaftliche Disziplin gegeben. So wird man ja auch nicht dadurch, daß man eine Sprache beherrscht, bereits zum Linguisten. Die Logik entstand durch die wissenschaftliche Reflexion auf das Schließen selbst. Obwohl auch das früh einsetzte, läßt sich sagen, daß die Logik als Wissenschaft erst von Aristoteles geschaffen wurde, der sie sogleich auf ein beträchtliches Niveau brachte. Seine Logik kann heute als ein Vorläufer der Prädikatenlogik und auch der Klassenlogik angesehen werden. Ein typischer Lehrsatz seiner Logik ist z. B.:

\subsection{Wenn $\mathbf{P}$ allen $\mathbf{M}$ und $\mathbf{M}$ allen $\mathbf{S}$ zukommt, so kommt auch $\mathbf{P}$ allen $\mathbf{S} \mathrm{zu}$.}

Die Termini P, M, S stehen dabei für Begriffe oder Klassenausdrücke.

Einen anderen Höhepunkt der antiken Logik stellt die von der Philosophie der Stoa geschaffene stoische Logik dar. Hauptvertreter im Hinblick auf die Logik waren Zenon von Kition, Diodoros Chronos und Chrysippos. Die Stoische Logik kann als Vorläufer der Aussagenlogik angesehen werden. Ein typischer Lehrsatz ist z. B.:

0.2 Wenn das Erste so das Zweite. Nun nicht das Zweite. Also nicht das Erste.

Die Ausdrücke das Erste, das Zweite stehen dabei für ganze Aussagesätze. Jedoch ist das oft mit den aristotelischen Termini vermengt worden.

In den folgenden zwei Jahrtausenden war die Logik so sehr von Aristoteles geprägt, daß sich andersartige Ansätze kaum durchsetzen konnten und auch keine bahnbrechenden Weiterentwicklungen erfolgten. Auch die stoische Logik fand nicht die Beachtung, die sie verdiente. Die meisten Schriften der stoischen Logiker gingen verloren und es sind nur (zumeist unfreundliche) Kommentare erhalten, aus denen man die stoische Logik erst rekonstruieren muß. Als man den hohen Rang der stoischen Logik erkannte, war die moderne Aussagenlogik bereits geschaffen und die stoische Logik nur noch von historischem Interesse. Übrigens lief das, was heute als "Logik" bezeichnet wird, lange unter dem Titel "Dialektik". Die Bezeichnung "Logik" hat sich erst im neunzehnten Jahrhundert endgültig durchgesetzt.

Eine Blütezeit erlebte die Logik in der Scholastik, als die antike Wissenschaft für das Abendland wiederentdeckt wurde. Dabei dominierte die Lehre des Aristoteles. Es wurden die Benennungen. für die Syllogismen und ein ausgeklügeltes mnemotechnisches System von Merksprüchen entwickelt. Die "Summulae Logicales" von Petrus Hispanus (später Papst Johannes XXI.) wurden für drei Jahrhunderte das führende Lehrbuch der Logik. Die Scholastiker befaßten sich viel mit semantischen Fragen. In dem bekannten Universalienstreit ging es um die Seinsweise der Allgemeinbegriffe, ob sie eine eigenständige Existenz haben (Realismus) oder nur Wörter sind (Nominalismus).

Viele Ideen des Neubeginns der Logik lassen sich sodann auf Gottfried Wilhelm Leibniz zurückführen. Er bemühte sich insbesondere um eine kalkülmäßige Erfassung 
der Logik. Es gelang ihm aber nicht, eine adäquate formale Sprache zu finden.

Die Logik galt, als die anderen Wissenschaften sich von der Autorität des Aristoteles lösten und dann begannen, sich stürmisch zu entwickeln, weithin als eine ziemlich abgeschlossene, aber recht sterile Disziplin.

Das änderte sich erst im neunzehnten Jahrhundert. Seit dieser Zeit wurde die Logik zu einem beträchtlichen Teil von Mathematikern weiterentwickelt. Dabei erfuhr sie eine tiefgreifende Umgestaltung. Die Methoden sind durchweg mathematisch und auch die Inhalte weitgehend auf die Mathematik bezogen.

Dabei ist die ältere Logik aber nicht zugunsten der modernen Logik aufgegeben worden, so wie es mit der Alchimie beim Aufkommen der Chemie der Fall war. Sie ist auch nicht in der Weise berichtigt worden, wie es mit der älteren Physik und Astronomie bei der Entwicklung der modernen Wissenschaft geschah. Die ältere Logik, die völlig korrekt war, ist in der modernen Logik aufgegangen, etwa so wie die antike Mathematik in der modernen Mathematik aufgegangen ist.

Charakteristisch für die moderne Logik ist die Verwendung künstlicher, formaler Sprachen anstelle natürlicher Sprachen. Das führt zu der Unterscheidung zwischen (formalen) Objektsprachen, die das Objekt der Untersuchung sind, und der Metasprache, deren inhaltliches Verständnis vorausgesetzt wird und in der die Untersuchung erfolgt. Hierdurch wird die präzise Definition logischer Begriffe und die Verwendung mathematischer Methoden erst ermöglicht.

Die Formalisienung geht in der Logik über das in der Mathematik übliche Maß insofern hinaus, als auch Wörter und Wendungen wie z. B.:

0.3 nicht, und, oder, wenn ..., so ..., ...genau dann, wenn..., für alle, es gibt ein, gehört zu

durch Symbole dargestellt werden, nämlich:

$0.3 \mathrm{a} \neg, \wedge, \vee, \Longrightarrow, \Longleftrightarrow, \forall, \exists, \in$

Die Aussage 0.1 dieses Paragraphen kann damit, unter Benutzung einer Variablen v, in folgender Weise formalisiert werden:

$0.1 \mathrm{a} \quad \forall \mathrm{v}(\mathrm{v} \in \mathrm{M} \Longrightarrow \mathrm{v} \in \mathrm{P}) \wedge \forall \mathrm{v}(\mathrm{v} \in \mathrm{S} \Longrightarrow \mathrm{v} \in \mathrm{M}) \Longrightarrow \forall \mathrm{v}(\mathrm{v} \in \mathrm{S} \Longrightarrow \mathrm{v} \in \mathrm{P})$

Und der Schluß in 0.2 kann formal so dargestellt werden:

$\begin{aligned} & 0.2 \mathrm{a} \begin{array}{l}\mathrm{p} \Longrightarrow \mathrm{q} \\ \neg \mathrm{q}\end{array} \\ & \neg \mathrm{p}\end{aligned}$

Es sollen nun noch einige Stichworte zur Entwicklung der modernen Logik gegeben werden, selbst wenn diese dem Leser u. U. nur andeutungsweise verständlich sind.

Die semantische Definition des Folgerungsbegriffs durch Betrachtung aller Uminterpretationen der Sprache findet sich bereits bei Bernhard Bolzano. Allerdings sind die 
sprachlichen Mittel bei ihm noch nicht präzisiert und er betrachtet nicht den Unterschied von logischen und nichtlogischen Konstanten.

George Boole versuchte, in die Logik ein Rechnen nach Art der Algebra einzuführen. Wenn die Variablen $x, y, \ldots$ für Begriffe (Klasssen) oder auch für Aussagen und,+ • für Vereinigung und Durchschnitt (bei Klassen) bzw. für "oder" und "und" (bei Aussagen) stehen, so erhält man logische Gesetze, die in vieler Hinsicht den Gesetzen des Zahlenrechnens ähneln, doch gibt es auch Unterschiede. Es gilt z. B. bei dieser logischen Interpretation:

$$
x \cdot(y+z)=(x \cdot y)+(x \cdot z) \text { und ebenso } x+(y \cdot z)=(x+y) \cdot(x+z)
$$

Noch heute redet man von der Booleschen Algebra, die insbesondere in der Informatik von Bedeutung ist.

Charles S. Peirce entwickelte sehr ausgereifte logische Systeme. Die Dualitiät zwischen dem einschließenden Oder und dem Und wurde von ihm klar formuliert, und er hatte auch schon praktisch die Prädikatenlogik in Händen. Seine Schriften blieben aber lange Zeit weitgehend unbekannt.

Ähnlich ging es zunächst Gottlob Frege, dem bedeutendsten Logiker des neunzehnten Jahrhunderts. Von ihm stammt die erste umfassende Formalisierung von Aussagenlogik, Prädikatenlogik, Kennzeichnungslogik, sowie der logischen Theorie der Klassen, Relationen und Funktionen. Seine Formalisierung umfaßt nicht nur die Sprache, auch die zugelassenen Schlußweisen sind genau angegeben. Frege gab die erste wirkliche Zahldefinition, d.h. er definierte den Zahlbegriff mit Hilfe rein logischer Begriffe und leitete die arithmetischen Gesetze logisch ab. Ferner leistete er bedeutende und heute sehr beachtete Beiträge zur Sprachphilosophie, insbesondere mit seiner Unterscheidung von Sinn und Bedeutung eines Ausdrucks. Freges unmittelbare Wirkung war aber gering. Dazu trug seine (zweidimensionale) Symbolik bei, die sich nicht durchsetzen konnte und die auf den ersten Blick sehr unübersichtlich wirkt. Ein tragischer Punkt war, daß kurz vor Erscheinen des letzten Bandes seines Hauptwerkes (Grundgesetze der Arithmetik, I 1893, II 1903) von Bertrand Russell darin ein Widerspruch entdeckt wurde (die sog. Russellsche Antinomie), der gar nicht so einfach ausgeräumt werden kann. Man kann die Antinomie etwa in der Weise formulieren, daß man die Klasse aller Klassen bildet, die sich nicht selbst als Element enthalten und dann fragt, ob diese Klasse sich selbst als Element enthält.

Inzwischen begann in der Mathematik mit der Entwicklung der Mengenlehre eine andere Entwicklungslinie. Die Mengenlehre ermöglichte die moderne axiomatische Methode. Letztlich ist durch diese Entwicklung die Mathematik völlig umgestaltet worden.

Die von Georg Cantor begründete und zu einem wesentlichen Teil selbst entwickelte Mengenlehre verstand sich zunächst gar nicht als ein logisches System, ist aber in der weiteren Entwicklung mit der Logik verschmolzen. Auch in der Mengenlehre tauchten Antinomien auf. Doch ließ sich Cantor, der nicht formal vorging, dadurch nicht beunruhigen. Eine Konsolidierung fand die Mengenlehre durch eine Axiomatisierung, die 1908 von Ernst Zermelo angegeben wurde.

Unabhängig von Frege befaßten sich auch Richard Dedekind und Guiseppe Peano mit dem Zahlbegriff. Sie charakterisierten die natürlichen Zahlen durch innere Eigenschaften, die die Nachfolgerfunktion und die Methode der vollständigen Induktion 
betreffen. Heute würde man sagen, daß sie die natürlichen Zahlen axiomatisch charakterisierten. Peano ging mehr formal vor und entwickelte eine (lineare) Symbolik, aus der die heute übliche logische Symbolik hervorging. David Hilbert legte 1899 eine Axiomatisierung der Geometrie vor. Diese hat die Entwicklung des modernen axiomatischen Standpunkts eingeleitet und auch die Semantik der modernen Logik beeinflußt. Frege hat allerdings dieser Sichtweise nichts abgewinnen können.

Bertrand Russell, der direkt durch Frege beeinflußt worden war und der die von ihm selbst entdeckte Antinomie ausräumen wollte, entwickelte die sog. Typenlogik, deren Grundidee ist, daß die mathematischen Objekte in verschiedene Stufen eingeteilt sind und eine Klasse jeweils von höherer Stufe als ihre Elemente ist. Er schrieb zusammen mit Alfred North Whitehead das monumentale Werk der "Principia Mathematica" (I 1910, II 1912, III 1913), das den eigentlichen Durchbruch brachte. Fortan war allgemein anerkannt, daß die Logik in der Form eines formalen Systems entwickelt werden sollte, das die sprachlichen Mittel und die zugelassenen Beweismittel umfaßt (was Frege zuerst realisiert hatte), und daß man dabei im Hinblick auf mögliche Antinomien kritisch vorgehen muß.

Russell war aber noch naiv-optimistisch bezüglich der Reichweite der formalen Methoden. Er wollte nachweisen (ähnlich wie schon vorher Frege mit seinem System der "Grundgesetze"), daß sich alle mathematischen Wahrheiten in seinem System der "Principia" beweisen lassen.

Die Überwindung der Antinomien war das zentrale Thema der Logik in den ersten drei Jahrzehnten des zwanzigsten Jahrhunderts. Luitzen J.E. Brouwer entwickelte ab 1908 die intuitionistische Mathematik, die in rigoroser Weise bereit war, Wesentliches vom klassischen Bestand der Mathematik zu opfern, wobei auch bedeutende Mathematiker, wie z. B. Hermann Weyl, dieser Richtung zuneigten. Das rief Hilbert auf den Plan, der erklärte, die Mathematik ließe sich "nicht aus dem von Cantor geschaffenen Paradies vertreiben". Er entwickelte das Programm der Beweistheorie, um durch den formalen und "finiten" Nachweis der Widerspruchsfreiheit den Bestand der klassischen Mathematik zu retten.

Der in den zwanziger Jahren recht heftig geführte Grundlagenstreit und die Sorgen um Widersprüche sind abgeflaut. Aber die Probleme sind nicht eigentlich gelöst worden. Nach wie vor gibt es verschiedene Grundlagenstandpunkte.

Der klassische Standpunkt verwendet in der Metatheorie Mengenlehre. Danach sind die abstrakten mathematischen Objekte "an sich" da, und ihre Eigenschaften werden "entdeckt". Er entspricht dem mittelalterlichen Begriffsrealismus und wird im Hinblick auf die Ideenlehre Platons auch als Platonismus bezeichnet.

Die Konstruktivisten sind die Nachfahren der Nominalisten. Nach dem konstruktivistischen Standpunkt "erschafft" man durch mathematische Begriffsbildung die mathematischen Objekte, die man sich nicht als unabhängig existierend vorzustellen braucht - bei einem strengeren Standpunkt - nicht so vorstellen soll.

Heute sind viele Logiker aber nicht mehr geneigt, sich strikt an einen Standpunkt zu binden. Eher wählen sie den Standpunkt je nach der Art der vorzunehmenden Untersuchung aus (etwa ob sie mengentheoretisch oder beweistheoretisch ist).

Nach dem Erscheinen der "Principia Mathematica" setzte eine intensive Arbeit an den logischen Systemen ein. Die sehr umständliche Russellsche Typenlogik wurde durch 
Frank P. Ramsey, Kurt Gödel und Alfred Tarski vereinfacht. Als einfachste Teile wurden Aussagenlogik und Prädikatenlogik (Logik der ersten Stufe) herauspräpariert. Die axiomatische Mengenlehre wurde weiterentwickelt, formalisiert und in die Logik eingebaut.

Es zeigten sich aber auch die ersten Grenzen formaler Methoden. Thoralf Skolem wies 1922 auf die Existenz abzählbarer Modelle der (prädikatenlogisch formalisierten) Mengenlehre hin und zeigte 1933 die Nichtcharakterisierbarkeit der natürlichen Zahlen in der Prädikatenlogik. Diese Resultate waren von ihm durchaus kritisch gemeint. Doch machen gerade die sich darin ausdrückenden Beschränkungen die Prädikatenlogik zu einem nützlichen logischen Werkzeug. Gödel gelang es 1930, für die Prädikatenlogik nachzuweisen, daß die bekannten logischen Schlußregeln wirklich ausreichen, um alle Folgerungen zu ziehen; die Logik der ersten Stufe ist vollständig.

Ein Jahr später erfolgte eine tiefe Zäsur in der Logik, die den Abschied von der naiven Vorstellung bedeutete, man könne alles was gilt, auch formal beweisen. Gödel zeigte 1931 die prinzipielle Unvollständigkeit der Logik höherer Stufe und jeder axiomatisch aufgebauten Arithmetik sowie die formale Unbeweisbarkeit der Widerspruchsfreiheit. Diese Sätze, die Grenzen formaler Methoden aufzeigen, sind vielleicht die bedeutendsten logischen Resultate überhaupt.

Hierdurch verlor die Logik höherer Stufe, die Typenlogik, an Gewicht. Die Logik der ersten Stufe, die Prädikatenlogik, die bis dahin nur als ein Fragment der "eigentlich interessierenden" Logik höherer Stufe gegolten hatte, wurde aufgewertet und gewann in der Folge eine zentrale Stellung in der Logik.

Durch die Gödelschen Sätze wurde auch das Hilbertsche Programm der Beweistheorie in der ursprünglich intendierten Form als undurchführbar nachgewiesen. Doch gelang es Gerhard Gentzen 1936, mit einer Erweiterung des "finiten Standpunkts" die Widerspruchsfreiheit der axiomatischen Zahlentheorie nachzuweisen. Seitdem hat sich die Beweistheorie von der engen Hilbertschen Zielsetzung gelöst und ist zu einem umfangreichen und weit entwickelten Teilgebiet der Logik geworden.

Es erwies sich dabei als unabdingbar, zu klären, was algorithmisch überhaupt gemacht werden kann. Das führte im Jahr 1936 zu einer Präzisierung des Berechenbarkeitsund Entscheidbarkeitsbegriffs, die man wirklich als letztgültig und nicht mehr verbesserbar ansehen kann. Daran waren neben Gödel besonders Alonzo Church, Stephen C. Kleene, Alan M. Turing und Emil L. Post beteiligt. Church zeigte 1936 die Unentscheidbarkeit der Logik erster Stufe. Das besagt, daß es keinen wie auch immer gearteten Algorithmus gibt, um von beliebig gegeben Aussagen der Prädikatenlogik zu entscheiden, ob sie allgemeingültig sind. Hierdurch wird eine Grenze algorithmischer Methoden aufgezeigt. Die Untersuchungen, die an die Begriffe der Berechenbarkeit und Entscheidbarkeit anknüpfen, haben zur Entwicklung der Rekursionstheorie geführt.

Die von Zermelo begründete axiomatische Mengenlehre hat sich zu einem umfangreichen Teilgebiet der Logik entwickelt. Ergänzungen zur Axiomatik kamen von Abraham A. Fraenkel, Thoralf Skolem, Johann (John) von Neumann, Kurt Gödel und Paul Bernays. Gödel bewies 1939 die relative Widerspruchsfreiheit des Auswahlaxioms (AC), das Zermelo eingeführt hatte und dem wegen seines nichtkonstruktiven Charakters oft eine geringe Evidenz zugeschrieben wird, und der Kontinuumshypothese $(\mathrm{CH})$, 
die besagt, daß es zwischen der Anzahl der natürlichen Zahlen und der der reellen Zahlen keine weiteren (unendlichen) Anzahlen gibt. Cantor hatte sich vergeblich bemüht, $\mathrm{CH}$ zu beweisen. Die volle Unabhängigkeit von $\mathrm{AC}$ und $\mathrm{CH}$ wurde 1961 von Paul Cohen gezeigt und löste eine Fülle weiterer Resultate aus.

$\mathrm{Zu}$ den klassischen Gebieten der Logik gehört auch die Modelltheorie. Ein frühes Resultat über die Kardinalzahl von Modellen von Formelmengen wurde 1915 von Leopold Löwenheim bewiesen und 1920 von Skolem erweitert. Grundlegend ist eine berühmte Arbeit von Tarski, in der er die Nichtdefinierbarkeit des Wahrheitsbegriffes für eine Sprache in ihr selbst zeigte. Das Resultat stammt aus dem Jahr 1931, die Hauptveröffentlichung erschien 1936. Das führte zur Unterscheidung von Objektsprache und Metasprache und zur Entwicklung der (mengentheoretisch fundierten) Semantik, die den Rahmen für die Modelltheorie abgibt. Tarski hat große Impulse zur Entwicklung der Modelltheorie gegeben, in der mathematische Strukturen unter möglichst allgemeinen Gesichtspunkten, unter Einsatz logischer Hilfsmittel und insbesondere unter Verwendung formaler Logiksprachen untersucht werden. Ein grundlegender Satz der Modelltheorie ist der Endlichkeitssatz, den Anatoli I. Malcev 1936 bewies.

Eine wichtige Seitenlinie zu Modelltheorie und Mengenlehre ist die von Abraham Robinson begründete Nichtstandardanalysis, in der man u.a. das Umgehen mit unendlich kleinen Größen auf eine exakte Grundlage stellen kann.

Enge Beziehungen bestehen zwischen der Logik und der Informatik. Das ist bei der Rekursionstheorie evident, in der mit der Explikation des Berechenbarkeitsbegriffs auch ein wichtiger Grundbegriff der Theoretischen Informatik geklärt wird.

In der Informatik besteht aber generell ein besonderer Bedarf an formalen logischen Methoden und Theorien. Während es für die Mathematik ausreicht, daß Theoreme und Beweise formalisierbar sind (weil mathematische Beweise von Menschen gemacht, gelesen und verstanden werden), müssen wesentliche Teile der Informatik streng formalisiert sein (weil Programme zwar (meist) von Menschen gemacht, aber von Maschinen ausgeführt werden, die nur über sehr begrenzte Fähigkeiten verfügen, Sinnzusammenhänge zu erkennen und Fehler zu korrigieren).

Ein wichtiges Ziel ist es deshalb, Methoden zum Beweis der Korrektheit von Programmen zu entwickeln. Man möchte, ähnlich wie ein Mathematiker die Gültigkeit eines Satzes beweist, streng nachweisen können, daß ein vorgelegtes Programm auch wirklich immer das tut, was es soll, daß es einer bestimmten Spezifikation genügt. Einen einflußreichen Ansatz gab C. A. R. Hoare an.

Beim Logischen Programmieren schließlich möchte man sogar erreichen, daß die Spezifikation eines Problems das Programm ersetzt. Die Maschine soll, gestützt auf eine vorher eingegebene umfangreiche Wissensbasis aus Fakten und Regeln, selbst die Lösung finden. Wegen der oben erwähnten Unentscheidbarkeit der Logik, ist das nicht vollständig möglich. Deshalb sind auch pragmatisch wichtige Teillösungen von Interesse.

Das ist auch in der Disziplin der Künstlichen Intelligenz der Fall, wo man sich um die Entwicklung von Systemen bemüht, die Leistungen erbringen (z. B. beim Planen, Entscheiden, Reagieren, Handeln), für die der Mensch seine kognitiven Fähigkeiten einsetzen muß. Es ist dafür eine theoretische Durchdringung des Umfeldes nach Art 
der Logik erforderlich, wobei aber eher typisch philosophische als mathematische Begriffe zu analysieren sind.

Natürlich hat die Logik enge Bindungen zur Philosophie, aus der sie ja historisch stammt. In der letzten Zeit ist die philosophisch orientierte Logik wieder erstarkt.

Dazu hat wesentlich die Entwicklung der Modallogik beigetragen. Darin geht es um die Begriffe "notwendig" und "möglich". Die ersten Untersuchungen dazu stammen bereits von Aristoteles. Auch in der Scholastik hat man sich intensiv mit Modallogik befaßt. Die Modallogik wurde aber als schwierig und unklar angesehen. Als Alexandria christlich wurde, galt die Modallogik - so mokierten sich später islamische Gelehrte - als "Gefahr für das Christentum", und es durfte nur die assertorische Logik des Aristoteles gelehrt werden. In die moderne Logik wurde die Modallogik durch Clarence I. Lewis wieder eingeführt, doch hat sie Willard v. O. Quine scharfsinnig kritisiert. Wirklich konsolidiert wurde die Modallogik erst durch die von Saul Kripke und Stig Kanger entwickelte Semantik.

Dabei hat sich die Modallogik von dem Ziel, eine logische Analyse von Notwendigkeit und Möglichkeit zu geben, gelöst und ist von paradigmatischer Bedeutung für die Analyse sog. intensionaler Kontexte geworden. Mit der Theoretischen Linguistik trifft man sich in dem Bemühen, das Funktionieren von Sprache allgemein zu analysieren und $\mathrm{zu}$ verstehen. Bahnbrechende Arbeiten zum Verhältnis von formalen und natürlichen Sprachen unter Einschluß der Semantik stammen von Richard Montague.

In diesem Buch wird der klassische Grundlagenstandpunkt eingenommen, der in der Metatheorie Mengenlehre voraussetzt und der sich immer wieder als tragfähig erwiesen hat.

Nach der Aussagenlogik wird ausführlich die Prädikatenlogik entwickelt. Darauf folgt die knappe Darstellung einer Klassenlogik, die aber alle wesentliche Definitionen enthält. Schließlich wird ein Ausblick auf die Modallogik und weitere philosophisch relevante Logiksysteme gegeben.

Die prädikatenlogischen Sprachen sind vornehmlich zur Formulierung mathematischer Sachverhalte geeignet. Man könnte meinen, sie seien für die Philosophie nicht relevant und für Anwendungen außerhalb der Mathematik würden andere logische Systeme mit anderen Sprachen besser passen.

Es soll hier keineswegs behauptet werden, daß die Prädikatenlogik der logischen Weisheit letzter Schluß ist. In vieler Hinsicht sind die prädikatenlogischen Sprachen zu starr und ihre semantischen Grundannahmen in realen Situationen unzutreffend.

Aber die Prädikatenlogik kann durchaus eigenes Interesse beanspruchen. Sie ist noch sehr einfach, hat aber trotzdem große Ausdrucksfähigkeit und ein beträchtliches Anwendungspotential. Sie setzt einen Standard, an dem sich andere logische Systeme messen lassen müssen. Außerdem dürfte sie für erweiterte logische Systeme eine Art Basis und Ausgangspunkt darstellen. Jedenfalls wird man ohne gründliche Kenntnis der Prädikatenlogik auch keine besseren Logiksysteme entwickeln können. Deshalb sollte sie im Mittelpunkt einer Einführung in die Logik stehen. 